Bioscientia Medicina Volume 4, Issue 3, Page No: 34-44 Available online : www.bioscmed.com Bio Sc Med 4(3) :34-44

\title{
Herpes Zoster: Clinical Manifestation, Treatment, and Prevention
}

\author{
M. Izazi Hari Purwoko ${ }^{1 \#}$, Hari Darmawan ${ }^{1}$ \\ ${ }^{1}$ Dermatology and Venereology Department, Faculty of Medicine Sriwijaya University, Palembang, \\ Indonesia \\ \#Corresponding authors Email: m_izazi_hp@yahoo.com
}

\begin{abstract}
Herpes zoster (HZ, shingles, zoster) is a disease due to reactivation and multiplication of persistent varicella zoster virus (VVZ) after suffering from previous varicella with characteristics of dermatomal rashes, pain, and unilateral. Dermatoms most often involved in HZ lesions are thoracal, trigeminal, lumbal, and cervical. The first lesion is usually erythematous macule or papule, which then turns into vesicle, then to pustule and to become crust, and persists for 2-3 weeks. The main goal of antiviral therapy in HZ patients is to reduce the expansion, duration, and severity of rashes and pain in primary dermatomes; prevent the spread of $\mathrm{HZ}$ to other places; and prevent post-herpes neuralgia. One of the prevention strategies for $\mathrm{HZ}$ in the elderly and high-risk individuals is vaccination using the varicella zoster virus vaccine that is live zoster vaccine or recombinant zoster vaccine.
\end{abstract}

Keywords : Herpes zoster, clinical manifestation, treatment, prevention

\section{Introduction}

Herpes zoster (HZ) (shingles, zoster) is a disease with a characteristic rash and unilateral dermatomal pain distribution caused by reactivation and multiplication of endogenous varicella zoster virus (VZV), persisting in latent phase in sensory ganglia after previous episode of varicella. ${ }^{1,2}$

Several classical clinical manifestations include painful herpetiform clustered vesicles, with an erythematous base, which is limited in one or two dermatomal areas. HZ occuring in healthy, immunocompetent individuals will reach spontaneous resolution and disappear without interventions. HZ on children may be of lighter severity compared to adults. Patients over 50 years of age and immunocompromised individuals harbor higher 
risk of acquiring $\mathrm{HZ}$ with clinical manifestations of rash, pain, and severe complications, causing distress and reducing quality of life. ${ }^{3,4}$

Medications will be the most effective for patients having the risk of bearing long and severe symptoms, especially immunocompromised individuals and those over 50 years of age. In general, drug choices differ by immunity status and personal clinical manifestations. ${ }^{3,4}$

One of the preventive strategies of $\mathrm{HZ}$ in general population, especially elderly and high-risk groups, is to stimulate immunity agains VZV by vaccination. ${ }^{3}$

\section{Clinical Manifestation}

A few days before rash eruption, fever, malaise, myalgia, headache, pain and paresthesia around dermatom occur. Most often, constant or intermittent pain is experienced by those older than 60, which varies from superficial tingling or itching, burning, till lancinating pain. Onset of pain is 48-72 hours before the first rash appear. Some patients may experience zoster sine herpetic with the form of acute segmental neuralgia without skin lesions. ${ }^{1,4,6}$

The most common clinical manifestation is unilateral distribution of rash, usually limited to one or two dermatoms. The first lesion is usually erythematous macule or papule, which then 12-24 hours after turns into vesicle, then to pustule by the third day. After 7-10 days, the pustule dries off to become crust, and persists for 2-3 weeks. New lesions may appear for the first 1-4 days (sometimes up to 7 days). ${ }^{1}$ Classical rash appear as painful herpetifom grouped vesicles sitting on an erythematous base, and is limited to one or two dermatoms. ${ }^{3,6}$ Skin lesions usually heal without residual rash, but may turn into hypo/hyperpigmentation following inflammation or scarring. Skin rash will be more severe and longer in older individual; therefore it is less severe and shorter in pediatric population. ${ }^{1}$ Sometimes, reginal lymphadenopathy may occur. ${ }^{3}$

Clinical manifestations can highly vary. HZ most often occurs in dermatoms with the most number of varicella lesions. ${ }^{1}$ Dermatom related to HZ lesion are usually within centripetal distribution of the initial varicella lesion. During varicella, virus spreads from skin lesion to sensory nerve endings, then ascends to ganglion. Other hypothesis believes that the ganglion is infected by hematogen route during viremia phase of varicella, and 
reactivation stimulus occurs most often in these ganglions. ${ }^{3,6}$ Dermatoms most often involved in $\mathrm{HZ}$ lesions are thoracal, trigeminal, lumbal, and cervical. ${ }^{10}$

HZ oticus (geniculate zoster, zoster auris, Ramsay Hunt syndrome, Hunt syndrome) occurs with involvement of geniculatum ganglion. The classic appearance is otalgia with herpetiform vesicles in external auricular canal or tympanic membrane, with or without facial paralysis, auditory symptoms (hearing disturbance), and vestibular symptoms. Ramsay Hunt syndrome triad consists of HZ lesion on ear, ipsilateral facial palsy, and anesthesia in $2 / 3$ anterior of the tongue. This syndrome is caused by involvement of N. VII, IX, and $\mathrm{X}^{3}$

$\mathrm{HZ}$ involving the eyelids signifies the involvement of the first branch (ophthalmicus) of trigeminal nerve, and is named HZ ophthalmicus. This type is often experienced by the elderly. HZ lesions on the bridge of the nose (Hutchinson's sign) is a predictor of ocular involvement. On occasions, blepharoconjunctivitis, keratitis, uveitis, and secondary glaucoma may occur. ${ }^{3,6}$

The involvement of second branch (maxillaris) and third branch (mandibularis) of trigeminal nerve causes intraoral HZ lesions accompanied with tooth pain. Maxillary branch involvement causes lesions in ipsilateral cheek, upper and lower lid, side of nose, nose mucose membrane, nasopharyng, tonsils, upper teeth, palatum, uvula, and tonsilar fossa. Involvement of mandibular branch causes lesions on the side of the head, external ear, external auricular cannal, lower lip, base of mouth, buccal mucose membrane, and tongue. . $^{3,6}$

Disseminated HZ is a generalized eruption with more than 10-12 extradermatomal vesicles, 7-14 days after the onset of classic dermatomal HZ. Disseminated HZ indicate decreased cellular immunity with various causes, including malignancy, radiation therapy, cancer chemotherapy, organ transplantation, or long term corticosteroid medications. ${ }^{3}$ In the immunocompromised, new lesions may appear till up to 2 weeks, and viremia with disseminated rash may occur with complications involving skin necrosis, scar, and visceral organ involvement including lungs, liver, and brain. HZ patient with active HIV infection may experience recurrent $\mathrm{HZ}$ in the same dermatom or other dermatoms. $\mathrm{HZ}$ patients with AIDS may experience severe $\mathrm{HZ}$ with dissemination to the skin and visceral organs, including acyclovir-resistant VZV with verrucose or hyperkeratotic lesions. ${ }^{1,8,10}$ 
HZ complications don't happen often in healthy children and adults, and most commonly reserved to the immunocompromised. The frequently reported complication is post herpetic neuralgia (PHN), which is a persistent pain for more than 4 weeks after $\mathrm{HZ}$ onset, and occuring $60 \%$ in patients $>60$ years old. Some skin complications include secodary bacterial infection, scar, zoster gangrenosum, and cutaneous dissemination. Ocular complications include conjunctivitis, keratitis, corneal ulcer, iridocyclitis, glaucoma, and blindness. Some visceral complications include pneumonitis, hepatitis, esophagitis, gastritis, cystitis, and arthritis. Neurological complications involve meningoencephalitis, cranial nerve palsy, sensory loss, deafness, peripheral nerve palsy. ${ }^{1}$

\section{Diagnosis}

HZ diagnosis is mainly based on anamnesis and physical examinations, with focus on the characteristic location and rash appearance with local pain. ${ }^{6}$ Laboratory diagnosis confirmation is done on atypical $\mathrm{HZ}$ patients, visceral organ and central nervous system involvement, or zoster sine herpete. Some laboratory diagnosis confirmation involve Tzanck smear, polymerase chain reaction (PCR), direct immunofluorescence assay (DFA), skin biopsy, and culture.

Tzanck smear is a simple and affordable laboratory confirmation test, but it cannot differentiate VZV with other herpesviruses. PCR may detect VZV DNA in skin lesion samples and visceral organs. PCR and VZV IgG test with cerebrospinal fluid sample are the first line laboratory examinations on $\mathrm{HZ}$ patients with visceral organ and central nervous system involvement. Skin biopsy and PCR can assist diagnosis on zoster sine herpetic cases. ${ }^{3,6}$

\section{Treatment}

During acute phase, in order to subside local symptoms and hasten vesicular lesion drying, a patient may do a compress with sterile saline solution, Burowi solution, or lotio calamin, which is applied on the involved skin for 30-60 minutes, 4-6 times a day. Topical antiviral therapy is proven not effective, thus is not recommended. ${ }^{1,3,6,8}$

The main objective of antiviral therapy on HZ patients is to limit spread, duration, and severity of lesion and pain on primary dermatom, reduce distribution of $\mathrm{HZ}$ to other locations, and prevent PHN. ${ }^{1}$ 
Most complications are caused by prolonged replication and VZV spread from the involved ganglion. Therefore it should be prevented by quick and effective antiviral therapy. ${ }^{1}$ Nucleoside analogues such as acyclovir, famcyclovir, and valacyclovir, and pirophosphate analogue phoscarnet has been proven to be effective to treat VZV infection. Afterwards, cellular enzymes will transform acyclovir monophosphate to acyclovir triphosphate which inhibits DNA polymerase virus to stop virus DNA synthesis. ${ }^{1}$ Phoscarnet is an inorganic pirophosphate analogue which inhibits viral replication. Antiviral activity is brought forward by selective inhibition on DNA polymerase and reverse transcriptase pyrophosphate-binding site, but it doesn't influence cellular DNA polymerase. Phoscarnet antiviral activity doesn't require phosphorilation by timidine kinase, thus proven effective for acyclovir-resistant VZV.,

\section{Herpes Zoster on Immunocompetent Adolescents and Adults}

Antiviral therapy is recommended to reduce time of lesion healing, duration and severity of acute pain on adult patient. Medication is administered in $\leq 72$ hours after rash onset. Antiviral therapy is recommended, even for those with lesion onset of $>72$ hours, if new vesicles still emerge, any cutaneous lesions exist, disseminated visceral or neurological involvement is suspected, HZ subtype is opthalmicus or oticus, and all immunocompromised patients. ${ }^{1,3,9,11}$

Antiviral drug regimen recommended for immunocompetend adolescents and adults are: ${ }^{1}$

- Those <50 years old: symptomatic therapy; or oral famcyclovir 3dd $500 \mathrm{mg}$ for 7 days; or oral valacyclovir 3x1 gram for 7 days; or oral acyclovir $5 \times 800 \mathrm{mg}$ for 7 days.

- $\quad$ Those $\geq 50$ years old, and any age with cranial nerve involvement (for example, HZ ophthalmicus): oral famcyclovir 3x500 mg for 7 days; or oral valacyclovir $3 \times 1$ gram for 7 days, or oral acyclovir 5x800 mg for 7 days.

The choice of drug depends on the price of the medicine and frequency of dosages in a day. Famcyclovir and valacyclovir are prefered compared to acyclovir for oral therapy due to their pharmacological advantages, less frequency of administration in a day, and higher antiviral activity in the bloodstream. ${ }^{1,3,12}$ 
Ophthalmicus HZ is better treated alongside ophthalmologists to prevent ocular complications, especially when the eye is involved. ${ }^{8,9}$

\section{Herpes Zoster on Immunocompromised Patients}

Less severe $\mathrm{HZ}$ on immunocompromised patients or those with local $\mathrm{HZ}$ may be given oral acyclovir, famcyclovir, or valacyclovir. ${ }^{1,3,9}$ Treatment regimen for immunocompromised individuals involve: ${ }^{1}$

- Mild immunocompromise, involving HIV infection: oral famcyclovir 3x500 mg for 7-10 days; or oral valacyclovir 3x1 gram for 7-10 days; or oral acyclovir 5x800 mg 7-10 days.

- Severe immunocompromise: intravenous acyclovir $10 \mathrm{mg} / \mathrm{kg}, 3 \mathrm{x}$ daily for 7-10 days.

- Acyclovir-resistant (AIDS end stage): intravenous phoscarnet $40 \mathrm{mg} / \mathrm{kg} 3 \mathrm{x}$ daily till resolution.

\section{Herpes Zoster on Pregnant Women and Children}

Pregnant women with HZ should be considered to be treated as soon as possible, to hasten lesion healing and reduce intensity and length of pain period. The recommended antiviral therapy for pregnant women are oral acyclovir 5x800 mg/day for 7-10 days and oral valacyclovir $3 \times 100 \mathrm{mg} /$ day for 7 days. ${ }^{4,13,14}$ Famcyclovir isn't recommended for pregnant and breastfeeding women. ${ }^{14}$ Intravenous acyclovir therapy is given to pregnant women with $\mathrm{HZ}$ involving cranial nerves, ocular nerve, mucose membrane, hemorrhagic lesion, disseminated lesion, and pneumonitis. Intravenous dosage ranges from 10-15 $\mathrm{mg} / \mathrm{kg}$, or $500 \mathrm{mg} / \mathrm{m}^{2}$ every 8 hours, for 5-10 days. ${ }^{13,14}$

Herpes zoster doesn't occur often in children, and if it does, it is majorly mild. Antiviral therapy is recommended if complications are present, if they are immunocompromised, or new lesions keep emerging after 3 weeks course of disease. Acyclovir is given orally, with dosage $20 \mathrm{mg} / \mathrm{kg} / \mathrm{dosage}$ (maximum of $800 \mathrm{mg} /$ dosage), 5 times per day, for 5-7 days. For immunocompromised children with severe HZ, they are given intravenous acyclovir $10 \mathrm{mg} / \mathrm{kg}$ or $500 \mathrm{mg} / \mathrm{m}^{2}$ every 8 jam, for 7-10 days., ${ }^{4,15}$

\section{Anti-inflammatory Therapy}


Corticosteroid usage for $\mathrm{HZ}$ is still debated. Inflammation happening in sensory ganglia plays a role in PHN, thus for its prevention, one may be given corticosteroid during acute phase of HZ. Initiation of corticosteroid must be hand-in-hand with antiviral therapy. ${ }^{1,4,10,16}$ The duration of corticosteroid treatment should not exceed antiviral therapy. ${ }^{1}$ Combination of acyclovir and corticosteroid is recommended for healthy adult $\mathrm{HZ}$ patients with moderate - severe pain, if neurological symptoms are significant, or if central nervous system, Ramsay-Hunt syndrome, or ocular complications are involved. $3,4,9,10$

The studies about acyclovir and corticosteroid combination therapy reported various dosage, route of administration, and treatment period. Dworkin reported prednison usage at $60 \mathrm{mg} /$ day for 1 week, then tappered off to $30 \mathrm{mg} /$ day for 1 week, followed by $15 \mathrm{mg} /$ day for 1 week. $^{10,11}$ Murakami reported Ramsay-Hunt syndrome medication using acyclovir $250 \mathrm{mg}$ IV 3 times daily, or oral acyclovir 5x800 mg/day for 7 days, combined with intravenous or oral prednison $1 \mathrm{mg} / \mathrm{kg} / \mathrm{day}$ with two divided doses for 5 days, tappered off slowly and stopped in 10 days. Coulson reported treatment of Ramsay-Hunt syndrome using oral acyclovir $5 \times 200 \mathrm{mg} /$ day for 21 days, followed with oral prednison 1 $\mathrm{mg} / \mathrm{kg} /$ day for 14 days then tappered off to $10 \mathrm{mg} /$ day before stopped. ${ }^{4}$

\section{Treatment of Acute Pain in $\mathrm{HZ}$}

Most of HZ patients experience acute pain, and it is usually the most reported symptom. Analgetics are necessary as adjuvants to antiviral therapy. ${ }^{9}$ Choice and dosage of drug depends on pain severity, underlying diseases, and response to medication. ${ }^{1,9}$ Patients with HZ and mild pain may be given oral acetaminophen for 325-1000 mg 4-6 times per day, or nonsteroidal anti-inflammatory drugs (NSAIDs) like oral ibuprofen 400 mg 4- times daily. Acetaminophen and NSAIDs are the drugs of choice to control HZ acute pain. ${ }^{10,11,17}$ Healthy $\mathrm{HZ}$ patient reporting moderate - severe acute pain on presentation, or with risk factors for PHN (women, age over 50 years od, lesion count $>50$, location in cranial/sacral area, hemorrhagic lesion, or dermatomal pain during prodromal phase) are recommended to be given tricyclic antidepressant analgetic, anticonvulsant or opiod/non-opioid. ${ }^{9}$ If pain control is still inadequate, doctors may consider to administer nerve block or local anesthesia. ${ }^{1}$ 
Anticonvulsant medications gabapentin and pregabalin are reported to reduce 50\% of acute pain in HZ. ${ }^{17}$ Gabapentin is used with initial dosage $100-300 \mathrm{mg}$ before night sleep, and can be increased by $100-300 \mathrm{mg}, 3$ times daily until 3-7 days and pain is tolerable (maximum dosage of $3600 \mathrm{mg} /$ day). Pregabalin is used with initial dosage $25-$ $75 \mathrm{mg}, 1-2$ times daily, and can be increased by $75 \mathrm{mg}, 2$ times daily for 3-7 days until pain is tolerable (maximum dosage of $600 \mathrm{mg} / \mathrm{day}$ ). Side effects range from somnolence, dizziness, ataxia, and peripheral edema. ${ }^{1,10,11,16}$

Triclyclic anidepressants (amitriptiline, nortriptiline, and desipramine) can reduce 44-67\% HZ acute pain. ${ }^{1}$ Initial dosage is 10-25 mg before night sleep, and can be increased $10-25 \mathrm{mg}$ weekly until pain is tolerable (maximum dosage of $150 \mathrm{mg} /$ day). Side effects include somnolence, dry mouth, blurred vision, sedation, orthostatic hypotension, constipation, heart arrhythmia, and urinary retention., ${ }^{7,10,16,17}$

Opioid/non-opioid analgetics are reported to be effective to reduce acute HZ pain, some of the medications being oxycodone amd tramadol. Oxycodone is used with initial dosage of $5 \mathrm{mg}$ every 4 hours, and can be increased $5 \mathrm{mg} 4$ times daily every week until pain is tolerable (maximum of $120 \mathrm{mg} / \mathrm{day}$ ). Some reported side effects are nausea, vomiting, constipation, sedation, cognitive disfunction, bronchospasm, orthostatic hypotension, and itching. Tramadol is used with initial dosage of $50 \mathrm{mg}, 1-2$ times daily, and can be increased $50-100 \mathrm{mg} /$ day, given in two divided doses until pain is tolerable (maximum of $400 \mathrm{mg} /$ day; $300 \mathrm{mg} /$ day for age $>75$ years old). ${ }^{10,11,16}$

\section{Prevention of HZ:}

\section{Varicella Zoster Virus Vaccination}

The prevention of HZ aims to avoid reactivation and spread of latent VZV virus. One of the preventive strategies of $\mathrm{HZ}$ in the general population, especially the elderly and high-risk individuals is to stimulate immunity against $\mathrm{VZV}{ }^{3}$

In the year 2006, the US Food and Drug Administration (FDA) and the Centers for Disease Control and Prevention (CDC) recommended the higher potency live attenuated vaccine VZV (Zostavax®) to prevent $\mathrm{HZ}$ on immunocompetent non-pregnant population, aged $\geq 60$ years old, including those who have had previous $\mathrm{HZ}$ episode. It is given in a single subcutaneous dose on the deltoid area. ${ }^{7,18}$ Protection spans till 3 years. 
Several contraindications to this medication is those severely immunocompromised, pregnant women, and children. ${ }^{3,10,20}$

In the year 2018, CDC recommended immunization on adults using zoster vaccine:

- 2 doses of recombinant zoster vaccine (RZV) (Shingrix $\left.{ }^{\circledR}\right)$ 2-6 months apart, is used for those aged $\geq 50$ years old, regardless of previous $\mathrm{HZ}$ episode, or have received live zoster vaccine (ZVL) (Zostavax $\left.{ }^{\circledR}\right)$.

- 2 doses of RZV, 2-6 months apart for adults having received ZVL, minimal of 2 months after ZVL.

- Administration of one of RZV or ZVL for those aged $\geq 60$ years (RZV is preferred). ${ }^{3,20}$

\section{Varicella Zoster Immune Globulin (VZIG)}

The Centers for Disease Control and Prevention (CDC) recommended passive immunization using VZIG to prevent or modify clinical manifestation of VZV infection on high risk individuals, children, pregnant women, or immunocompromised individuals exposed to varicella or herpes zoster. VZIG is given intramuscullarly, with dosage of 125 $\mathrm{U} / 10 \mathrm{~kg}$ (maximum of $625 \mathrm{U}$; minimum $125 \mathrm{U}$ ) or $0,5-1 \mathrm{mg} / \mathrm{kg}$. The best effect is achieved if administered as soon as possible from exposure, and at least $\leq 96$ hours after exposure. Protection after VZIG lasts for around 3 weeks. ${ }^{3,7,10}$

\section{Conclusion}

Classic clinical characteristics of $\mathrm{HZ}$ are painful herpetiform grouped vesicles, sitting on top of an erythematous base, limited to one or two dermatoms. Skin rash is more severe and longer in the elderly, but milder and shorter in children. Dermatoms most often involved are thoracal, trigeminal, lumbal, and cervical. Clinical manifestations may vary.

$\mathrm{HZ}$ diagnosis is based on clinical appearance, and laboratory diagnosis comfirmation is done on atypical $\mathrm{HZ}$ patients.

$\mathrm{HZ}$ treatment is recommended for those with risk of experiencing long and severe symptoms, especially immunocompromised populations and those over 50 years old. Acyclovir, famcyclovir, valacyclovir, and phoscanet is proven to be effective to treat VZV infection. Medication is administered in $\leq 72$ hours after onset of rash. Combination 
therapy acyclovir and corticosteroid is recommended for acute moderate - severe pain, those experiencing significant neurological symptoms or with central nervous system manifestation, Ramsay-Hunt syndrome, and ocular complications.

Analgetic medications are given as adjuvants to antiviral therapy to treat acute pain in HZ. HZ patients with acute mild pain can be given acetaminophen or NSAIDs. HZ patients with moderate - severe pain may be given tricyclic antidepressants analgetic, anticonvulsant or opiod/non-opioid.

One of the strategies of prevention of HZ in the elderly and high-risk individuals is to vaccinate using zoster vaccine live or recombinant zoster vaccine. Passive immunization using VZIG is recommended to prevent or modify clinical manifestation of VZV infection on high-risk individuals, children, pregnant women, or immunocompromised exposed to varicella and herpes zoster.

\section{References}

1. Schmader KE, Oxman MN. Varicella and Herpes Zoster. In: Goldsmith LA, Katz SI, Gilchrest BA, Paller AS, Leffell DJ, Wolff K, editors. Fitzpatrick's Dermatology in General Medicine, $8^{\text {th }}$ ed. New York: McGraw Hill; 2012.p.2383401.

2. Sterling JC. Viral Infections. In: Griffiths CEM, Barker J, Bleiker T, Chalmers R, Creamer D, editors. Rook's Textbook of Dermatology, $9^{\text {th }}$ ed. John Wiley \& Sons, Ltd; 2016:25.24-31.

3. Janniger CK, Elston DM. Herpes Zoster. Medscape. 2019 [Updated 2019, jan 25]. Available at: https://emedicine.medscape.com/article/1132465.

4. Koshy E, Mengting L, Kumar H, Jianbo W. Epidemiology, treatment and prevention of herpes zoster: A comprehensive review. Indian J Dermatol Venereol Leprol; 2018; 84: 251-62.

5. Somayaji R, Elliott JA, Sibbald RG. Dermatologic Manifestations of Herpes Zoster. In: Watson CPN, Gershon AA, Oxman MN, editors. Herpes zoster: Postherpetic Neuralgis and Other Complikation. Focus on Treatment and Prevention. Cham, Switzerland: Springer International Publishing; 2017: 103-12.

6. Cohen JI. Herpes Zoster. N Engl J Med. 2013; 369(3): 255-63. 
7. Cohen KR, Salbu RL, Frank J, Israel I. Presentation and Management of Herpes Zoster (Shingles) in the Geriatric Population. P T. 2013;38(4):217-27

8. Werner RN, Nikkels AF, Marinovic B, Schafer M, Czarnecka-Operacz M, et al. European consensus-based (S2k) Guideline on the Management of Herpes Zoster guided by the European Dermatology Forum (EDF) in cooperation with the European Academy of Dermatology and Venereology (EADV), Part 2: Treatment. JEADV; 2017; 31: 20-9

9. Jhon AR, Canaday DH. Herpes Zoster in the Older Adult. Infect Dis Clin N Am. 2017;31:811-26

10. Gershon AA. Antiviral Therapy and Lokal Treatment for Herpes Zoster. In: Watson CPN, Gershon AA, Oxman MN, editors. Herpes zoster: Postherpetic Neuralgis and Other Complikation. Focus on Treatment and Prevention. Cham, Switzerland: Springer International Publishing; 2017: 97-102.

11. Hayward K, Cline A, Stephens A, Street L. Management of herpes zoster (shingles) during pregnancy. J Obstet Gynaecol; 2018;38(7):887-94.

12. Schafer R, Davis M, Philippi JC. Herpes Zoster in Pregnancy. J Midwifery Womens Health. 2019;64(2):230-35

13. Leung AKC, Baranklin B. Herpes Zoster in Childhood. OJPed.2015;5:39-44.

14. Haanpaa M, Rice ASC, Rowbotham MC. Treating Herpes Zoster and Postherpetic Neuralgia. Pain. 2015;23(4):1-8

15. Saguil A, Kane S, Mercado M, Lauters R. Herpes Zoster and Postherpetic Neuralgia: Prevention and Management. Am Fam Physician. 2017; 96(10): 65663.

16. Bader MS. Herpes Zoster: Diagnostic, Therapeutic, and Preventive Approaches. Postgrad Med. 2013 Sep;125(5):78-91.

17. Le P, Rothberg M. Herpes zoster Infection. BMJ 2019 [cited 2019, jan 25].;364: k5095:1-6.

18. Dooling KL, Guo A, Patel M, Lee GM, Moore K, Belongio EA, et al. Recommendations of the Advisory Committee on Immunization Practices for Use of Herpes Zoster Vaccines. MMWR Morb Mortal Wkly Rep. 2018;67(3):103-8. 